sclerosis, optic neuritis, transverse myelitis or NMO spectrum disorders ${ }^{1,2}$. Associated morbidity and mortality of each is vastly different. Gaining accurate information through appropriate investigation is vital for appropriate treatment and counselling. There is currently no national or international guideline for investigation of an acute demyelinating episode.

Methods Retrospective review of all cases of a first demyelinating event over a 10 year period $(2008$ - 2018) at Cork University Hospital. Laboratory investigations, imaging and clinic letters were reviewed.

Results In total eighteen cases were reviewed. Eventual diagnoses were 7 ADEM, 4 ADEM with transverse myelitis, 4 Multiple Sclerosis and 3 Optic Neuritis. Median presentation age was 6 years (1 year 4 months - 15 years 10 months). WCC and CSF microscopy was done in 100\%. CRP and ESR done in $89 \%$ and $28 \%$ respectively. Investigations for bacterial and viral causes either on serum, CSF or swabs was inconsistent varying between $11-83 \%$. CSF antibodies, including anti MOG, anti-NMDA, Aquaporin 4 and anti voltage gated potassium channel antibodies were sent in $6-39 \%$ of cases dependant on test. Oligoclonal bands were sent in $83 \%$. Imaging was undertaken in all cases with seventeen having an MRI Brain. Median time to MR brain was 1 day (0 days -6 days). Fourteen cases had a MR spine with median time to spinal imaging of 2 days (1 day - 11 days).

Conclusion This review highlights the variable approach to investigation of suspected demyelination. The wide differential and need for prompt treatment to prevent long term neurological disability means there are multiple complex investigations required within a short time period. The laboratory investigations and neuroimaging required are labour intensive and incur significant financial cost. This is of particular importance in children, many of whom will require sedation and at times general anaesthetic to ensure successful obtaining of samples. The availability of a local protocol would guide clinicians investigation when faced with an unfamiliar presentation under significant time pressure. It would ensure appropriate and timely investigation enabling appropriate treatment and counselling.

\section{GP232 MANAGEMENT OF SEIZURES IN CHILDREN WITH THERAPY-RESISTANT EPILEPSY}

\footnotetext{
1,2Globa Oksana Valer'yevna* ${ }^{*},{ }^{1,2}$ Liudmila Kuzenkova, ${ }^{1}$ Natalia Zhourkova, ${ }^{1}$ Elena Sorokina, ${ }^{1}$ Tatiana Podkletnova, ${ }^{1}$ Natalia Andreenko, ${ }^{1}$ Kiril Savost'yanov, ${ }^{1}$ Vsevolod Pinelis. 'National Medical Research Center for Children's Health, Moscow, Russian Federation; ${ }^{2}$ Sechenov university, Moscow, Russian Federation
}

\subsection{6/archdischild-2019-epa.291}

Epilepsies in children represent a heterogeneous group of disorders and syndromes with different etiology, severity, prognosis and treatment. Early diagnosis, accurate recognition of underlying aetiologies leads to more effective management and treatment and improve overall health and quality of life. Genetic testing is very important in the cases of therapy-resistant seizures. The purpose of study was to recognise the possible reason of failed AED treatment and to find the ways to owercome it.

Methods 45 patients with different forms of epilepsy aged from 3 months to 16 years not the candidate for surgical treatment have been studied. The long duration EEG, high resolution MRI, blood biochemical tests, blood level lactate and ammonia, amino acid, organic acid and disturbance of fatty oxidation by TMS, genetic investigations (mtDNA and exome sequence), measurement of autoantibodies to NR2 and GluR1 in blood serum by ELISA were performed to these children.

Results The respiratory chain disorders confirmed by mtDNA sequence were found in 11 children. Metabolic epilepsies discovered in patients have the following origins: two with glutaric aciduria type1, one - glutaric aciduria type2, one with propionic aciduria, one with methylmalonic aciduria, one with Gaucher's disease type3, two patient with glycogenosis type 9, two patients with ceroid lipofuscinosis type 2 and 6,lysosomal storage disorders in 3 cases.

Genetic epilepsies with mutation in genes SCN8A (two patients), GRIN2A, KCNMA1, SRPX2, SCN9A, ACO2, ARHGEF9, 15q11.2q13.3,TSC- 4 patients were revealed. In other cases with normal MRI the reason of pharmacoresistant seizures was not discover yet. The elevated level of autoantibodies to glutamate NR2 and GluR1 receptors were found in children of these groups. But in patients with metabolic epilepsies the elevation level of autoantibodies to NR2 was in 4 to 7 times higher in comparing with children with genetic epilepsies. In children with metabolic disorders and energy metabolism disorders we use the specific therapy (special diet, L-carnitine, vitamins, enzyme replacement therapy etc) in cases which it possible, avoid valproic acid in treatment of children with mitochondrial disorders and glutaric acidurias, as well we use the phenytoin in patient with potassium channel mutation SCN8A. These treatment management led to reduction in seizures frequency or even to seizures remission in some cases.

Conclusions The recognition and diagnostic of underlying etiologies of intractable seizures improve the treatment management in many cases. The excessive NMDA transmission might be the part of pathogenesis of seizures in children with inborn error of metabolism.

\section{GP233 LIFESAVING MECHANICAL THROMBECTOMY IN PAEDIATRIC STROKE}

${ }^{1}$ Emily Farnan*, ${ }^{1} \mathrm{MP}$ O'Riordan Stephen, ${ }^{2}$ Noel Fanning, ${ }^{2}$ Gerald Wyse, ${ }^{3}$ Clodagh Ryan, ${ }^{4}$ Niamh Mc Sweeney. ${ }^{1}$ Department of Paediatrics and Child Health, Cork University Hospital, Cork, Ireland; ${ }^{2}$ Interventional Neuroradiology, Cork University Hospital, Cork, Ireland; ${ }^{3}$ Department of Paediatric Haematology, Mercy University Hospital, Cork, Ireland; ${ }^{4}$ Paediatric Neurology, Cork University Hospital, Cork, Ireland

10.1136/archdischild-2019-epa.292

Introduction Childhood arterial ischaemic stroke (AIS) is uncommon with a reported incidence between 1.2 and 7.91 per 100,000 per year. ${ }^{1,2,3}$ Previously it was thought that children with AIS had a good outcome due to brain plasticity; however, mortality has been reported in up to $28 \%$, and morbidity in up to $70 \%$ of survivors. ${ }^{4,5}$ There are no randomised trials of mechanical thrombectomy in children. The 2017 published RCPCH stroke guidelines draw on the excellent outcomes for mechanical thrombectomy in adult trials and recommend referral for intra-arterial clot extraction in patients with NIHSS score of 6 or more and up to 12 hours post onset if there is salvageable brain tissue on imaging. ${ }^{6}$ There are only 29 paediatric cases published in the literature that have undergone mechanical thrombectomy, 12 of which were for posterior circulation AIS. 